\title{
Synchrotron-radiation photoemission and x-ray absorption of Fe silicides
}

\author{
Fausto Sirotti \\ Laboratoire pour l'Utilisation du Rayonnement Electromagnetique, Université de Paris-Sud, F-91405 Orsay, France \\ and Laboratorium fur Festkoerperphysik, ETH, CH-8093 Zurich, Switzerland \\ Maurizio De Santis \\ Laboratoire pour l'Utilisation du Rayonnement Electromagnetique, Université de Paris-Sud, F-91405 Orsay, France \\ and Dipartimento di Fisica dell "Universita" dell'Aquila, Coppito I-67010, Italy \\ Giorgio Rossi \\ Laboratoire pour l'Utilisation du Rayonnement Electromagnetique, Université de Paris-Sud, F-91405 Orsay, France \\ and Laboratorium fur Festkoerperphysik, ETH, CH-8093 Zurich, Switzerland
}

(Received 8 February 1993)

\begin{abstract}
The valence-band and core-electron states of the iron silicides $\mathrm{FeSi}, \alpha-\mathrm{FeSi}_{2}$, and $\beta$-FeSi ${ }_{2}$, were measured by synchrotron-radiation photoemission and $\mathrm{x}$-ray absorption. The analysis of the $\mathrm{Fe} 3 s, \mathrm{Fe} 3 p$, and Si $2 p$ core-level photoemission line shape is made by comparing the silicide data with the data for bcc $\mathrm{Fe}$, hcp $\mathrm{Co}$, fcc $\mathrm{Cu}, \mathrm{CoSi}_{2}$, and $\mathrm{Si}(111) 7 \times 7$ surfaces. The $\mathrm{Fe} 3 p$ spin-orbit splitting in silicides is resolved spectroscopically. The results are discussed in connection with the electronic properties of the silicides and also provide reference standards for the very active spectroscopic research on the metastable interface-silicide phases of iron.
\end{abstract}

\section{INTRODUCTION}

Stoichiometric Fe silicides as well as metastable interface phases can be produced by solid-state epitaxy on silicon substrates. The electronic properties of iron silicides are very different as a function of stoichiometry and structure: cubic FeSi ( $B-20$ structure) is a narrow gap $(0.05 \mathrm{eV})$ semiconductor, ${ }^{1,2}$ orthorhombic $\beta-\mathrm{FeSi}_{2}$ has a band gap of $0.85 \mathrm{eV}, 3,4$ and tetragonal $\alpha-\mathrm{FeSi}_{2}$ is a metal. All these phases can be found at iron-silicon interfaces when attempting epitaxial growth of silicides. ${ }^{5-7}$ A large research effort has been stimulated by the theoretical possibility of controlling the epitaxial growth of the silicides on silicon single-crystal substrates thus obtaining semiconductor-semiconductor heterojunctions or metalsemiconductor Schottky junctions. In particular, one attempts to grow homogeneous films of an epitaxial constrained metastable iron-disilicide phase with the cubic fluorite $\left(\mathrm{CaF}_{2}\right)$ structure: band-structure calculations ${ }^{8}$ predict, for such a hypothetical $\gamma-\mathrm{FeSi}_{2}$ phase, metallicity and ferromagnetic ordering. A large body of this research is based on electron spectroscopies. ${ }^{9-12}$ Amorphous $\mathrm{Fe}-\mathrm{Si} / \mathrm{Si}$ multilayers display ferromagnetic behavior and are widely studied because of the low coercivity and their thickness-dependent in-plane versus perpendicular magnetic ordering. ${ }^{13}$

We have studied in detail the line shape of $\mathrm{Fe} 3 p, \mathrm{Fe} 3 s$, and $\mathrm{Si} 2 p$ core levels of the bulk silicides in order to provide better understanding of these spectra and therefore a basis for fingerprinting the growth of interfaces with photoelectron spectroscopy. We discuss the spectroscopic analysis of the core-level data with respect to long-range parameters such as metallicity and magnetism of $\mathrm{Fe}$ - silicide interfaces. The occupied and empty $3 d$ band states are measured by photoemission and soft x-rayabsorption spectroscopy, respectively.

\section{EXPERIMENT}

Atomically clean $\mathrm{FeSi}$ and $\alpha-\mathrm{FeSi}_{2}$ surfaces were obtained by grinding with diamond file polycrystalline rods in an ultrahigh vacuum (UHV) $\left(2 \times 10^{-10} \mathrm{mbar}\right)$. Cu $K \alpha$ $\mathrm{x}$-ray powder diffraction on the rod material showed that the FeSi sample was single phase. The quenched $\alpha-\mathrm{FeSi}_{2}$ samples, produced from batches, contained $\mathrm{FeSi}$ as a minority phase involving about $15 \%( \pm 5 \%)$ of the $\mathrm{Fe}$ atoms. This is a typical result of batch preparations due to the iron-defective nature of the tetragonal $\alpha$ phase of $\mathrm{FeSi}_{2}$. The UHV scraping technique produces clean surfaces without macroscopic annealing of the sample, nor atom-selective effects: it warrants maintaining the sample stoichiometry, including the same proportions of minority phases as present in the bulk material.

$\beta-\mathrm{FeSi}_{2}$ was grown in situ by reactive deposition of $\mathrm{Fe}$ onto an atomically clean $\mathrm{Si}(111) 7 \times 7$ surface maintained at $600^{\circ} \mathrm{C}$ by flowing a direct current through the silicon wafer. $\mathrm{Fe}$ was evaporated from an $e$-beam evaporator in a vacuum better than $1 \times 10^{-9} \mathrm{mbar}$ at a rate of $5 \AA / \mathrm{min}$ as controlled by a quartz microbalance. The sample temperature was monitored by an optical pirometer, its absolute value is quoted with a $\pm 50^{\circ} \mathrm{C}$ error bar. The spectroscopic study of the samples was done in situ, as discussed below in detail. A subsequent crystallographic study was done ex situ on a four-circle diffractometer with synchrotron $x$ rays, ${ }^{14}$ exploring in-plane and indepth Bragg diffraction in the case of the epitaxial $\beta$ - 


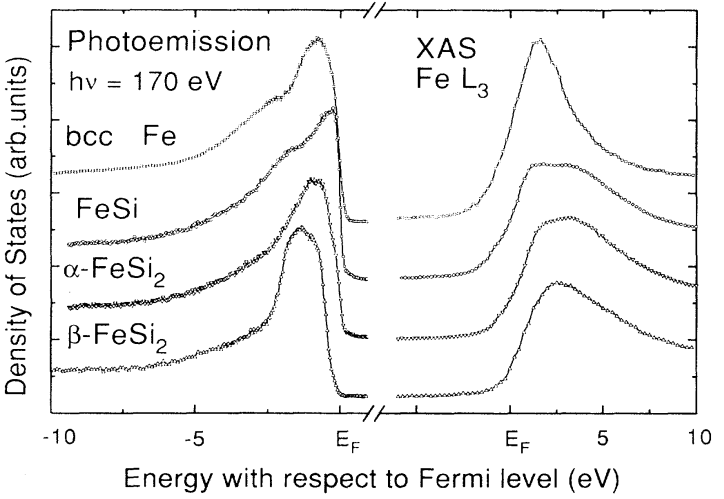

FIG. 1. Occupied and unoccupied density of states of bce-Fe, FeSi, $\alpha-\mathrm{FeSi}_{2}$, and $\beta-\mathrm{FeSi}_{2}$. In the left panel are shown the photoemission spectra measured with a photon energy of $170 \mathrm{eV}$. In the right panel are presented the $\mathrm{Fe} L_{3}$-edge spectra representing the empty $3 d$ states.

$\mathrm{FeSi}_{2}$ sample. Reference polycrystalline bcc Fe films of 100-200- $\AA$ thickness were produced in situ by the same deposition procedure onto sputtered Ta substrates, giving identical results as obtained from an Ar-sputtered bulk polycrystalline $\mathrm{Fe}$ sample of nominal 99.999 purity (Johnson Matthey Puratronic). Ondulator radiation from the DOMINO insertion device mounted on the SuperAco positron storage ring at Orsay was monochromatized by means of a $10-\mathrm{m}$ toroidal grating monochromator equipped with a 800 lines/mm grating for $140<h v<400$ $\mathrm{eV}$ photon-energy output and with a 1800 lines $/ \mathrm{mm}$ grating for $350<h v<900 \mathrm{eV}$ photon energies with resolution power of 1000. Absolute energy calibration of the 1800 lines/mm grating was not possible during these experiments so that the $\mathrm{x}$-ray-absorption spectroscopy (XAS) data analysis is limited to the line shape and $L_{2,3}$ branching ratios. Angle-integrated photoemission spectra were obtained with a Riber MAC-II electrostatic electronenergy analyzer whose axis makes an angle of $40^{\circ}$ to the linear polarization vector of the synchrotron light beam, and lies along the sample surface normal. The overall energy resolution is quoted for the various spectra. The XAS spectra were measured with $45^{\circ}$ incidence of the $x$ rays by collecting the total electron yield with a polarized electron multiplier, as well as by directly measuring the sample current. In these conditions the XAS spectra are not surface sensitive. All data were acquired within $2 \mathrm{~h}$ after sample preparation in a vacuum of $2 \times 10^{-10} \mathrm{mbar}$. Several preparations were made for each sample (scrapings, epitaxial growth) yielding consistent results.

\section{RESULTS}

Figure 1 presents on the left panel the spectra representative of the one-hole density of states of the valence band, as obtained by angle-integrated photoemission, and on the right panel the one-electron spectra of the empty conduction-band states, as obtained by $L$-edge x-ray absorption, for $\mathrm{FeSi}, \alpha-\mathrm{FeSi}_{2}, \beta-\mathrm{FeSi}_{2}$, and for polycrystalline bcc Fe. The photoemission spectra were obtained with $h v=170 \mathrm{eV}$, i.e., with a high cross section for the $\mathrm{Fe} 3 d$ states relative to $\mathrm{Fe} 4 s p$ and to $\mathrm{Si} 3 s p$ states, and represent the energy distribution of the occupied part of the $3 d$ bands. The absorption spectra on the right side of Fig. 1 were aligned on the Fermi level of polycrystalline bec iron.

$\mathrm{Fe} 3 s$ and $3 p$ spin-integrated data from bcc $\mathrm{Fe}$ are presented in Fig. 2 along with reference spin-resolved spectra from the literature. The data for the iron silicides are collected in Fig. 3 ( $3 p$ levels) along with reference spectra for fcc $\mathrm{Cu}$ and for $\mathrm{CoSi}_{2}$, and in Fig. 4 ( $3 \mathrm{~s}$ levels). The data points were fit by Lorentzian-Gaussian and Doniach-Sunjic ${ }^{24}$ functions, and by a proportional background function. The parameters obtained from the fit are reported in Table I. The data for the silicon $2 p$ core levels, measured with comparable final-state energy as the Fe core levels, are collected in Fig. 5. The fitting procedure was calibrated on the $\operatorname{Si}(111) 7 \times 7$ surface, obtaining the well-established rest atom and adatom peaks at, respectively, negative and positive shifts with respect to the substrate $\mathrm{Si}$ peak as summarized in Table II. A surface-shifted component is present for all silicides. Fig-
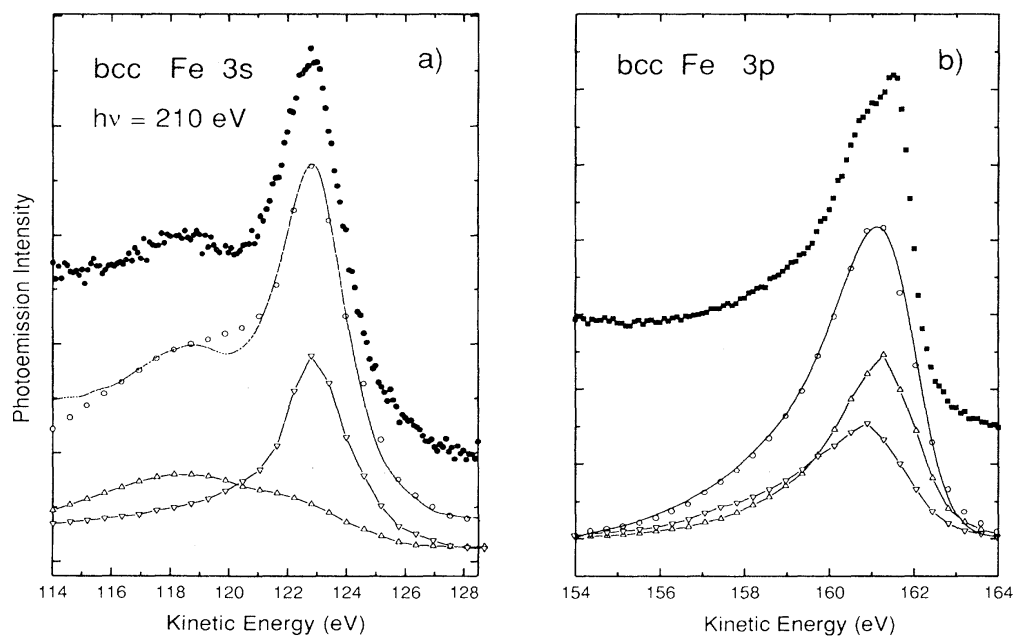

FIG. 2. Comparison of our spin-integrated data of $\mathrm{Fe} 3 s$ and $3 p$ core levels in bcc $\mathrm{Fe}$ (top curves) with the spin-resolved measurements from Refs. 20 and 22 (bottom). The curves in the middle are our broadened spectra (continuous) compared with the sum of the spinresolved components (points). 

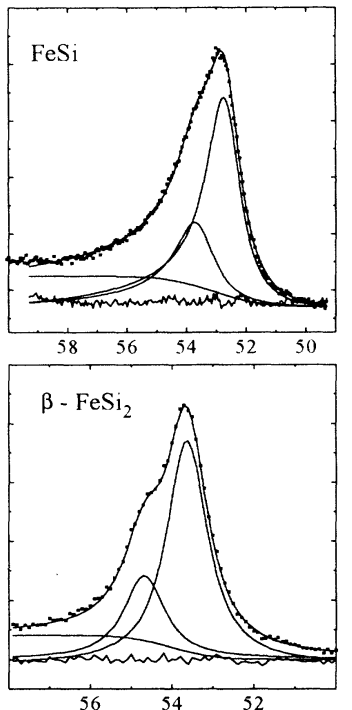
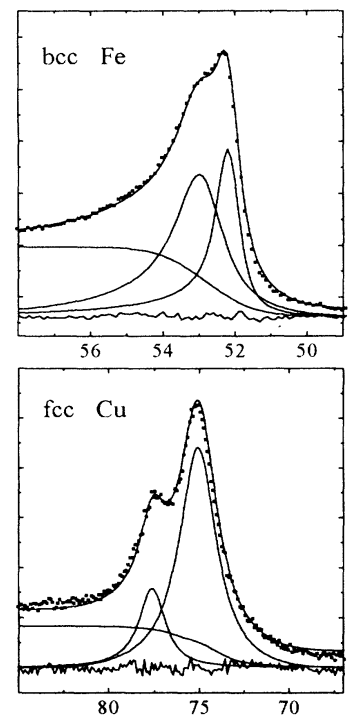
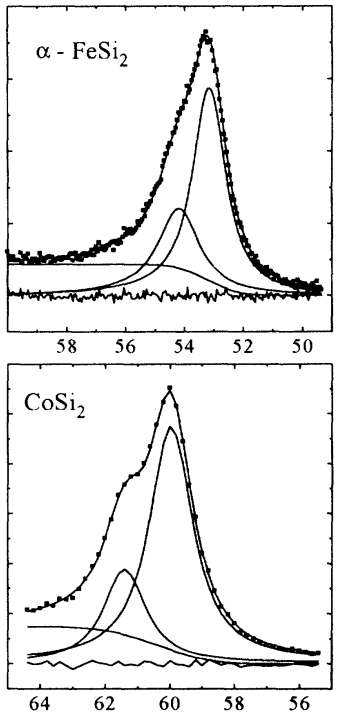

Binding Energy (eV)

FIG. 3. Photoemission from the $3 p$ core levels $(h v=170 \mathrm{eV})$ in bcc $\mathrm{Fe}, \mathrm{FeSi}, \alpha-\mathrm{FeSi}_{2}$, epitaxial $\beta-\mathrm{FeSi}_{2}, \mathrm{CoSi}_{2}$, and fcc $\mathrm{Cu}$. Raw data (points) are shown along with the best-fit spectra (continuous), the corresponding components and the background. In the bottom of each panel is reported the residual difference between the data and the fit. The fit parameters are reported in Table $\mathbf{I}$.

TABLE I. Parameters obtained from the best fit of the $3 p$ core-level photoemission spectra of the silicides and of bcc iron, hcp cobalt, and fcc copper. The different columns report the following: the values of the low binding-energy peak $(\mathrm{BE})$, the energy shift of the higher binding-energy component, the Lorentzian width of the two peaks, and the asymmetry parameter. The Gaussian width was $0.38 \mathrm{eV}$ for all the fits, representing the experimental energy resolution.

\begin{tabular}{lccccl}
\hline \hline & BE & $\Delta E$ & $L_{1}$ & $L_{2}$ & Asymmetry \\
\hline bcc $\mathrm{Fe}$ & 52.1 & 0.77 & 0.6 & 1.2 & 0.2 \\
$\mathrm{FeSi}$ & 52.7 & 0.95 & 1.1 & 1.4 & 0.18 \\
$\beta-\mathrm{FeSi}_{2}$ & 53.6 & 1.04 & 1.1 & 1.1 & 0 \\
$\alpha-\mathrm{FeSi}_{2}$ & 53.1 & 1.04 & 1.2 & 1.6 & 0.025 \\
$\mathrm{CoSi}_{2}$ & 60.0 & 1.43 & 1.5 & 1.5 & 0 \\
$\mathrm{hcp} \mathrm{Co}$ & 59.0 & 1.03 & 1 & 1.5 & 0.15 \\
$\mathrm{fcc} \mathrm{Cu}$ & 75.0 & 2.5 & 2.3 & 2.1 & 0.025 \\
\hline \hline
\end{tabular}

TABLE II. Parameters obtained from the best fit of the Si- $2 p$ core-level photoemission spectra for the silicides and for $\mathrm{Si}(111) 7 \times 7$. The binding energy of the different components is given along with their relative intensity (percent of the total intensity), the Lorentzian widths, and the asymmetry parameter. The Gaussian width was $0.38 \mathrm{eV}$ for all the fits, representing the experimental energy resolution.

\begin{tabular}{lccll}
\hline \hline & BE & Intensity (\%) & L.W. & Asymmetry \\
\hline $\mathrm{Si}(111) 7 \times 7$ & 99.1 & 74 & 0.13 & 0 \\
& 99.4 & 22 & 0.13 & 0 \\
$\mathrm{FeSi}$ & 98.4 & 4 & 0.13 & 0 \\
& 99.0 & 69 & 0.13 & 0.05 \\
$\beta-\mathrm{FeSi}_{2}$ & 98.6 & 31 & 0.13 & 0.05 \\
& 99.7 & 69 & 0.18 & 0 \\
$\alpha-\mathrm{FeSi}_{2}$ & 99.3 & 31 & 0.1 & 0 \\
& 99.2 & 67 & 0.18 & 0.025 \\
\hline \hline
\end{tabular}




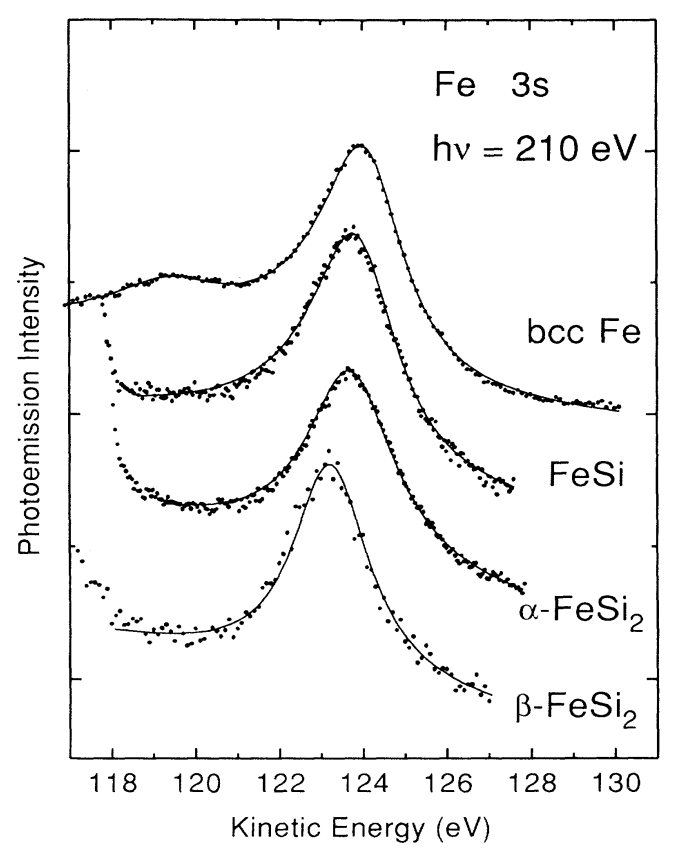

FIG. 4. Fe-3s core-level spectra $(h v=210 \mathrm{eV})$ of bcc $\mathrm{Fe}$, $\mathrm{FeSi}, \alpha-\mathrm{FeSi}_{2}$, and $\beta-\mathrm{FeSi}_{2}$. The continuous line is the best fit obtained with a Gaussian width of $0.38 \mathrm{eV}$ and a Lorentzian of $2.2 \mathrm{eV}$.
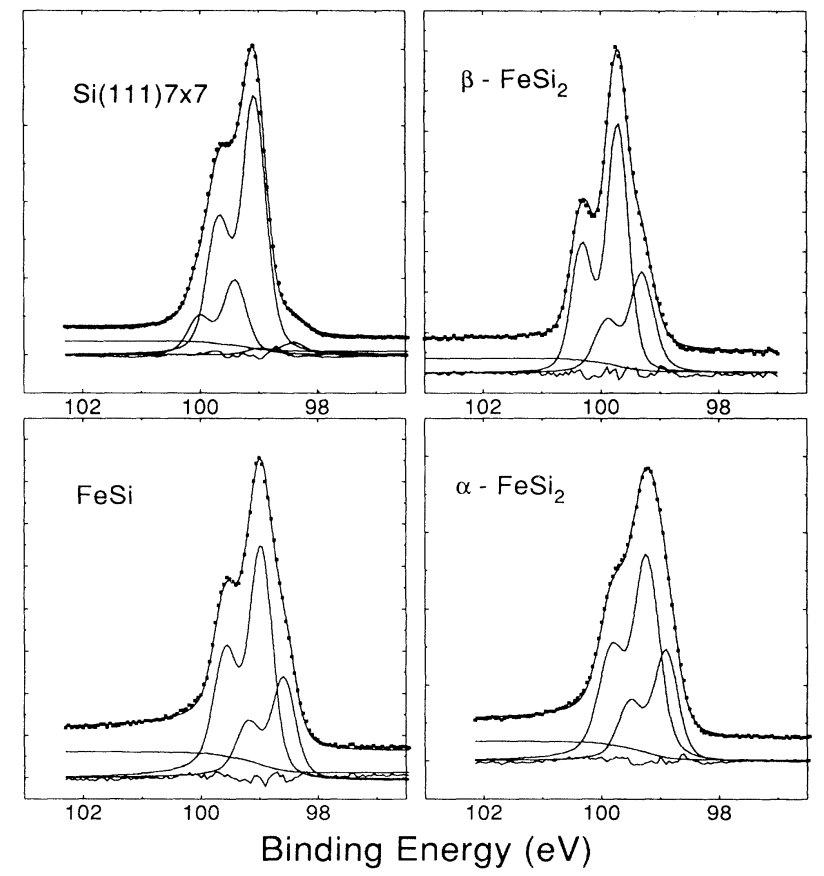

FIG. 5. Photoemission spectra of the $\mathrm{Si}-2 p$ core level $(h v=170 \mathrm{eV})$ from $\mathrm{Si}(111) 7 \times 7, \mathrm{FeSi}, \alpha-\mathrm{FeSi}_{2}$, and epitaxial $\beta$ $\mathrm{FeSi}_{2}$. Raw data (points) are shown with the best fit and the corresponding components and background. In the bottom of each panel is reported the residual difference between the data and the fit. The fit parameters are reported in Table II.

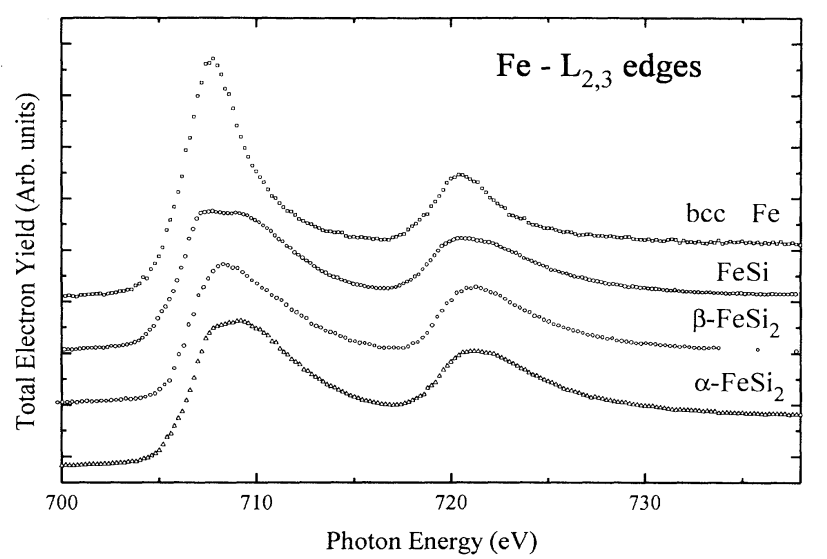

FIG. 6. X-ray-absorption spectra at the $\mathrm{Fe}-L_{2,3}$ edges of bcc-Fe, $\mathrm{FeSi}, \alpha-\mathrm{FeSi}{ }_{2}$, and epitaxial $\beta-\mathrm{FeSi}_{2}$ on silicon.

ure 6 presents the total electron yield $L_{2,3}$ absorption spectra for the silicides and for bcc Fe: the absorption branching ratio is seen to be strongly reduced in the silicides.

\section{DISCUSSION}

\section{Density of electron states}

The bonding structure of transition-metal silicides has been widely studied both spectroscopically ${ }^{15}$ and theoretically by means of band-structure ${ }^{16}$ and total-energy ${ }^{17}$ calculations. The silicide bonding is based on the rehybridization of the transition-metal $d$ bands with the silicon $s p^{3}$ bands giving, in the case of metallic silicides, a peak of bonding states well below the Fermi level and antibonding states crossing the Fermi level and extending several $\mathrm{eV}$ above it. ${ }^{16}$ The photoemission in the proximity of the Fermi level is very intense in FeSi (see Fig. 1). The shoulder at $-1.8 \mathrm{eV}$ below $E_{F}$ indicates the peak of the bonding Fe $d-$ Si $p$ hybrid states. The prominent peak at -0.3 $\mathrm{eV}$ is due to a localized $\mathrm{Fe} d$ band. FeSi is a nonmagnetic narrow gap $(50 \mathrm{meV})$ semiconductor. ${ }^{18}$ The correlation of the $d$ states in the localized band is reflected in the line shape of the core levels, as discussed below: no valenceband satellites are measured though, in agreement with resonant photoemission studies. ${ }^{2}$ A recent $\mathrm{Si} L_{2,3}$ soft-xray emission study ${ }^{13}$ showed that the peak of silicon states hybridized with the $\mathrm{Fe} d$ states lies at about $-2 \mathrm{eV}$ below the Fermi level confirming the bonding nature of the states that contribute to the shoulder in the photoemission spectrum. The empty part of the $d$ density of states (DOS) (in the presence of a $2 p$ core hole) is probed by $L_{3}$-edge XAS: the spectrum has a first peak at $1.8 \mathrm{eV}$ and a shoulder at $4 \mathrm{eV}$ above the inflection point of the edge. Configuration interaction is probably responsible for the large width of the XAS peak which probes both the empty localized $d$ states and the hybrid antibonding states.

The $\alpha-\mathrm{FeSi}_{2}$ raw spectra are presented in the third panel of Fig. 1: they contain about 15\% of signal from 
the minority $\mathrm{FeSi}$ phase. The $\alpha-\mathrm{FeSi}_{2}$ photoemission show a clear Fermi edge even after substraction of the scaled spectra for the FeSi impurity phase. The main peak lies at $-0.9 \mathrm{eV}$ and extends down to $-5 \mathrm{eV}$. It represents the hybrid $d-p$ bonding states of the disilicide. The empty $d$ states show a weak metallic edge and a smooth double-peak structure centered at $2.7 \mathrm{eV}$ above the edge corresponding to antibonding hybrid states.

The DOS of the epitaxial $\beta-\mathrm{FeSi}_{2}$ phase is markedly different: the $\mathrm{Fe} d$-Si $s p$ hybrid occupied states form a rather symmetric main peak with a fine structure centered at $-1.4 \mathrm{eV}$ and an extrapolated band edge at -0.2 $\mathrm{eV}$ from the Fermi level. The bandwidth and shape closely resemble the $d$-partial DOS calculated by Christensen. ${ }^{8} \mathrm{X}$-ray emission results for $\beta$-FeSi ${ }_{2}$ (Ref. 13) also confirm the hybridized nature of the electron states of the whole $d$ band. The spectrum of the empty $3 d$ states show a sharper peak, with respect to the metallic tetragonal phase, at about $2.5 \mathrm{eV}$ above the edge.

The main difference between the electron states of $\beta$ $\mathrm{FeSi}_{2}$ with respect to $\alpha-\mathrm{FeSi}_{2}$ is, therefore, a wider energy gap between bonding and antibonding $d$-hybrid bands, with the consequent lack of partially occupied antibonding states near the Fermi level. The semiconducting gap of $\beta-\mathrm{FeSi}_{2}$ cannot be established by the comparison of photoemission and $\mathrm{x}$-ray absorption due to the core-hole effect on the absorption energy; nevertheless, the overall energy distribution of the empty $d$ states allows to complete the picture of the $d$-band hybridization scheme.

\section{Core-levels photoemission}

The line shape of the $\mathrm{Fe} 3 s$ and $3 p$ core levels in bcc-Fe has been long debated: ${ }^{19}$ their understanding has recently improved thanks to four independent spin-resolved photoemission experiments carried out on pure Fe films and single-crystal surfaces. ${ }^{20-22}$ These studies show that the valence-band exchange splitting in ferromagnetic $\mathrm{Fe}$ has a direct influence on the core-level photoemission line shape: a fully polarized satellite peak is measured in the $3 s$ photoemission $^{20}$ and two largely overlapping exchange-split multiplets are measured in $3 p$ photoemission. $^{22}$ The conclusions on the exchange-interaction nature of the core-level satellites cannot be generalized for other ferromagnets: recent resonant spectroscopy results on fcc $\mathrm{Ni}$ show that the large asymmetry in the $3 p$ peak and the satellite of the $3 s$ peak can be understood in terms of $d$ mixing, i.e., configuration interaction within the highly correlated narrow $d$ band of $\mathrm{Ni}^{23}$ We compare our spin-integrated, but better energy resolved, $\mathrm{Fe} 3 \mathrm{~s}$ and $3 p$ data, with the spin-resolved data: a simple fit of the line shapes can be obtained as shown in Fig. 2. Our best fit of the measured $3 p$ line shapes is shown in Fig. 3: it was obtained by using Gaussian and Lorentzian peaks along with the asymmetry parameter introduced by Doniach and Sinjic. ${ }^{24}$ A step background following the integral of the peak was subtracted. The Gaussian width $(0.38 \mathrm{eV})$ was taken as a measure of the experimental resolution and was kept fixed in the fitting procedure for all the spectra of a given core level.

\section{Fe $3 p$ photoemission}

The $3 p$ peak shape should reflect spin-orbit interaction, exchange splitting, and $d$-mixing effects (configuration interaction in the $d$ bands when $d$ - $d$ correlation is high). All of these effects contribute to the intense tail of the $3 p$ spectrum of bcc Fe: a metallic energy loss and DoniacSunjic spectrum alone cannot explain neither the width nor the intensity of the tail. In the spectrum of $\mathrm{Cu} 3 p$ the Doniach-Sunjic effect amounts to only a small asymmetry. By comparison with the results of the spinresolved experiment by Sinkovic and co-workers, ${ }^{22}$ one can associate the main part of the low-energy tail of the $3 p$ peak to a broad majority spin multiplet, and the leading peak to the minority spin multiplet. The bcc-Fe $3 p$ line shape is therefore dominated by exchange splitting, the spin-orbit splitting being mixed in and not retrievable from the data. The large difference in the Lorentzian width that approximates the shape of the two multiplets may be related to an effective longer lifetime for the minority spin $3 p$ holes that can be filled only by the scarce minority spin electrons of the spin-split valence band. We stress that although the fitting of the bcc Fe $3 p$ photoemission peak was constrained by the spin-resolved data, the final result of Fig. 3 represents the approximate envelope of two final-state multiplets which depend on the combination of spin-orbit, exchange, and correlations; a deeper understanding of the spectra would require further experiments, e.g., the temperature dependence, and is beyond the scope of this work on silicides. Consistent line-shape parameters for the $3 p$ core levels of ferromagnetic hcp Co, as measured in the same conditions, are given in Table I.

The $\mathrm{Fe} 3 p$ peak in FeSi lies at $52.9 \mathrm{eV}$ : it can be decomposed in a doublet showing an energy splitting of $0.95 \mathrm{eV}$, a branching ratio of less than 2, a marked asymmetry and a slightly higher Lorentzian value for the lower energy peak than for the higher binding-energy component. The peak asymmetry is surprisingly large, of the same order as for bcc Fe: it cannot be connected to metallicity since FeSi is a narrow gap semiconductor nor to exchange interaction. ${ }^{18}$ The Fe site in FeSi has silicon neighbors at distances between 2.29 and $2.52 \AA$ and six iron neighbors at $2.75 \AA$. $^{11}$ Correlations within the localized $d$ band and configuration interaction are probably large in $\mathrm{FeSi}$ : a resonant photoemission experiment on the core-level line shape could shed light on this point.

The $\mathrm{Fe} 3 p$ peak in $\alpha-\mathrm{FeSi}_{2}$ lies at $53.3-\mathrm{eV}$ binding energy. The line shape is composed of a simple doublet with unequal widths, energy splitting of $1.05 \mathrm{eV}$, and a weak asymmetric tail approximated by a Doniach-Sunjic factor of 0.025 which can be attributed to the metallic character of the $\alpha$ disilicide. Fe occupies a single site in the tetragonal $\alpha-\mathrm{FeSi}_{2}$ and is coordinated to 8 silicon neighbors at $2.35 \AA$ and $8 \mathrm{Fe}$ neighbors, four at $2.68 \AA$ and four at 3.79- $\AA$ distance. ${ }^{11}$

The $\mathrm{Fe} 3 p$ peak in $\beta-\mathrm{FeSi}_{2}$ lies at $53.7-\mathrm{eV}$ binding energy, i.e., $0.5-\mathrm{eV}$ higher binding energy than in $\alpha-\mathrm{FeSi}_{2}$. The line shape is drastically simplified: a clear splitting of the doublet is measured with identical Lorentzian linewidths for both peaks which are symmetric. The en- 
ergy splitting of the two peaks is of $1.05 \mathrm{eV}$ which we attribute to spin-orbit splitting. The $3 p$ branching ratio is nonstatistical. Fe atoms in $\beta-\mathrm{FeSi}_{2}$ occupy two nearly cubic sites ${ }^{25}$ with silicon first neighbors at $2.34-2.39 \AA$ (site 1) and 2.34-2.44 $\AA$ (site 2). The very similar firstneighbor distances and bond angles imply a very small difference in binding energy for the two sites, which cannot be resolved in our experiment; $\mathrm{Fe}-\mathrm{Fe}$ second nearestneighbors occur between 2.96 and $4.05 \AA$. The large energy difference measured for the $\mathrm{Fe} 3 p$ photoemission of $\alpha-\mathrm{FeSi}_{2}$ and $\beta-\mathrm{FeSi}_{2}$ does not correspond to large differences in the atomic environment and should be attributed to screening effects.

In Table I and Fig. 3 the results for the iron silicides are compared with the $3 p$ core-level line shape of $\mathrm{CoSi}_{2}$, a metallic silicide having the $\mathrm{CaF}_{2}$ structure, i.e., a single Co site at the center of a cube of silicon nearest neighbors at $2.31 \AA$, and a long Co-Co second-neighbor distance of $3.8 \AA$. A consistent result is found: a doublet of identical Lorentzian widths, spin-orbit splitting of $1.43 \mathrm{eV}$, and a nonstatistical branching ratio. The metallic character of this disilicide contributes a very small asymmetry.

\section{Fe $3 s$ photoemission}

The satellite peak in the $3 s$ core-level photoemission from bcc $\mathrm{Fe}$ has $100 \%$ spin polarization. ${ }^{20}$ The different final-state energy of the $3 s$ core photoelectrons depends on the spin orientation of the core hole left behind and on its interaction with the spin-split $3 d$ band. According to this result the absence of the $3 s$ satellite in an iron compound would indicate the lack of magnetic ordering. More generally, the absence of a $3 s$ satellite indicates a large rehybridization of the $d$ bands with respect to bcc $\mathrm{Fe}$. The raising intensity at about 6-eV lower kinetic energy in the silicide Fe $3 s$ spectra is due to the foot of the very intense $\mathrm{Si} 2 p$ photoemission peak: this reduces the energy range for analyzing the $\mathrm{Fe} 3 s$ peak shape. It is nevertheless, clear that the prominent $3 s$ satellite of bcc$\mathrm{Fe}$ is absent in the silicides. ${ }^{19}$

The $3 s$ line shape of FeSi can be fit by a single peak with 2.2-eV Lorentzian width and a DoniachSunjic-type asymmetry of 0.18 , identical to that obtained in the fit of the $\mathrm{Fe} 3 p$ core levels. A previous study showed that the line shape is independent of temperature. ${ }^{1}$ The $3 s$ spectrum of $\alpha-\mathrm{FeSi}_{2}$ shows the same fit by a single peak with $2.2-\mathrm{eV}$ Lorentzian width and a reduced asymmetry of 0.02 ; finally, the $3 s$ spectrum of $\beta-\mathrm{FeSi}_{2}$ is fit by a single, symmetric peak of 2.2-eV Lorentzian width. The final-state energies of the Fe $3 s$ peak of $\alpha$ $\mathrm{FeSi}_{2}$ and $\beta-\mathrm{FeSi}_{2}$ differ by about $0.5 \mathrm{eV}$, likewise the $\mathrm{Fe}$ $3 p$ spectra. The photoemission intensity between the $\mathrm{Fe}$ $3 s$ and $\mathrm{Si} 2 p$ peaks in $\mathrm{FeSi}$ is therefore due to the same phenomena that originate the large asymmetry of the $3 p$ peaks, i.e., a consequence of correlations within the $d$ bands. The metallic screening explains the weaker asymmetry of the $3 s$ peak of $\alpha-\mathrm{FeSi}_{2}$.

\section{The Si $2 p$ core photoemission}

As a reference line shape we take the complex, but well understood, $2 p$ line shape of a $\operatorname{Si}(111) 7 \times 7$ surface. Our decomposition parameters are listed in Table II and are fully consistent with the recent literature. ${ }^{26}$ The spectrum of FeSi shows a main peak at 99-eV binding energy and a prominent shoulder at lower binding energy which can be understood as a surface termination of the silicide. The environment of silicon in the bulk FeSi sites is due to iron atoms at distances between 2.29 and $2.52 \AA$ and six silicon atoms at 2.78- $\AA$ distance. The large intensity of the second silicon peak measured at lower binding energy indicates that silicon termination stabilizes most if not all of the surface orientations since the FeSi sample is polycrystalline. This is equivalent to say that $\mathrm{Si}$ enrichment occurs at the grain boundaries.

The Si $2 p$ line shape of $\alpha-\mathrm{FeSi}_{2}$ is centered at $99.2-\mathrm{eV}$ binding energy. It shows a broad peak with a skewed shape towards lower binding energy. The intensity of the low binding-energy tail can be explained by the minority FeSi phase contribution. A spectra subtraction of approximately $15 \%$ FeSi line shape does not modify the main peak nor the higher binding-energy side of the peak. The silicon environment in $\alpha-\mathrm{FeSi}_{2}$ is due to four iron neighbors at close distance of $2.35 \AA$, one silicon neighbor at $2.36 \AA$, and five silicon neighbors between 2.68 and $2.77 \AA$. ${ }^{11}$ The presence of four strong Fe-Si bonds accounts for the chemical shift of about $100 \mathrm{meV}$ towards higher binding energies with respect to bulk silicon. The Fe-defective stoichiometry of the $\alpha$ phase and the presence of the minority FeSi phase are reflected in the width of the spectrum.

The Si $2 p$ photoemission of $\beta-\mathrm{FeSi}_{2}$ shows a bulk peak at $99.7 \mathrm{eV}$ and an extended shoulder to the lower binding energies. Two kinds of nearly tetrahedrical silicon sites exist in $\beta-\mathrm{FeSi}_{2}$, each one coordinated with four $\mathrm{Fe}$ neighbors between 2.33 and $2.43 \AA$ and six Si neighbors at distances of about $2.55 \AA .{ }^{25}$ The Si environment is similar to that of the $\alpha$ phase, yet the measured binding energy differs by $0.5 \mathrm{eV}$. This is similar to the effect on the $\mathrm{Fe} 3 p$ and $3 s$ photoemission and is probably due to the different screening of the core hole in the photoemission final state in the metallic versus semiconducting disilicides. Likewise, the case of the $\mathrm{Fe}$ inequivalent sites the energy difference between the two silicon sites is small and not resolved in the photoemission.

Silicon surface termination effects are known in the fluorite-structure disilicides $\mathrm{CoSi}_{2}, \mathrm{NiSi}_{2}$, and in epitaxial $\mathrm{FeSi}_{2}$ on $\mathrm{Si}(100){ }^{12}$ Table II also contains the data of the deconvolution of silicon terminated $\mathrm{CoSi}_{2}$ for reference. The total relative intensity of surface versus bulk $2 p$ photoemission in the silicides is larger than in pure silicon. This is a further confirmation of the termination of the silicides with a silicon overlayer.

\section{Fe $L_{2,3}$ x-ray-absorption branching ratio}

The $\mathrm{Fe} L_{2,3}$ absorption edges correspond to resonant transitions from $2 p$ core states to the empty $3 d$ band states, or equivalently to the conversion of spin-polarized (in $\mathrm{Fe}$ ) or spin-unpolarized (in the silicides) $3 d$ valence holes into $2 p$ core holes. The $L_{2,3}$ branching ratio in XAS of transition metals and transition-metal silicides often deviates from the statistical value. In the $5 d$ and $4 d$ 
periods the spin-orbit term in the $d$ band is large enough to allow a discussion of the XAS in terms of $L S$ coupling and therefore recognize the spin-orbit character of the empty-state multiplets. Unequal filling of the spin-orbit split $d$ band gives a reasonable explanation of the nonstatistical branching ratios. Examples are the $\mathrm{Pt} L_{2,3}$ branching ratio in PtSi versus fcc Pt (Ref. 27) and the Pd $L_{2,3}$ edges in $\mathrm{PdSi}$ and $\mathbf{P d}_{2} \mathrm{Si}$ versus fcc Pd. ${ }^{28}$ Also, electrostatic interactions in the final state can explain the anomalous branching ratios: a rationale of this widely observed behavior has been given recently in Ref. 29 . The situation is less clear for the $3 d$ ferromagnetic solids: the branching ratio in $\mathrm{Ni}$ and $\mathrm{Fe}$ exceeds a value of 3 and no quantitative understanding of this result is available insofar. Empirically, it is found that the high-spin $\mathrm{Fe}$ compounds all have high branching ratios, while the low-spin Fe compounds have nearly statistical branching ratios. ${ }^{29}$ The results presented in Fig. 6 confirm the high branching ratio (3.1) for bcc $\mathrm{Fe}$ and show a very close to statistical branching ratio for $\mathrm{FeSi}, \alpha-\mathrm{FeSi}_{2}$, and $\beta-\mathrm{FeSi}_{2}$.

\section{CONCLUSIONS}

We have measured photoemission and XAS spectra of $\mathrm{FeSi}, \alpha-\mathrm{FeSi}_{2}, \beta-\mathrm{FeSi}_{2}$, and bcc $\mathrm{Fe}$. Binding energies and deconvolution parameters of the $\mathrm{Fe}$ and $\mathrm{Si}$ core-level peaks were given which are accurate results within our experimental resolution and fitting approximations. Key elements for the understanding of the line shapes of the disilicides were obtained: (1) we could retrieve directly from the disilicide spectra the true spin-orbit splitting for the Fe $3 p$ core subshell which is $1.05 \pm 0.05 \mathrm{eV}$; $(2)$ the metallic $\left(\alpha-\mathrm{FeSi}_{2}\right.$ and $\left.\mathrm{CoSi} \mathrm{i}_{2}\right)$ or semiconducting $\left(\beta-\mathrm{FeSi}_{2}\right)$ character of the silicides could be distinguished by the presence (or absence) of a small asymmetry in all corelevel peaks, with a Doniach-Sunjic-like fitting value comparable to that of fcc $\mathrm{Cu}$; (3) the strongly asymmetric shapes of FeSi core-level spectra are attributed to correlation in the narrow $d$ band; (4) the effects of exchange splitting on the core-level line shapes, which are very strong in ferromagnetic bcc $\mathrm{Fe}$ and hcp $\mathrm{Co}$, are clearly not found in the silicides; (5) the $L_{2,3}$ branching ratio in the XAS spectra of the silicides is statistical, as expected for nonmagnetic samples; and (6) the photoemission final-state energies for the iron disilicides are largely affected by screening depending on the metallic or semiconducting phase.

The surface of the silicides is silicon terminated for all polycrystalline $\alpha-\mathrm{FeSi}_{2}$ and $\mathrm{FeSi}$ and epitaxial $\beta-\mathrm{FeSi}_{2}$. These results provide reference standards for the photoemission line-shape analysis of other iron-silicon systems of great relevance: soft-ferromagnetic amorphous $\mathrm{Fe} / \mathrm{Si}$ alloys and $\mathrm{Fe} / \mathrm{Si}$ interfaces.

\section{ACKNOWLEDGMENTS}

Thanks are due to M. C. Sichére for the $\mathrm{Cu} K \alpha$ x-ray powder-diffraction analysis, and to A. Walhauer, $N$. Jedrecy, and $M$. Sauvage for the synchrotron $x$-ray diffraction on the epitaxial $\beta-\mathrm{FeSi}_{2}$ sample. X. Jin, O. Sakho, and W. Nuñes-Rodrigues helped with the experiments and are gratefully acknowledged. This work was partially supported by the Swiss National Fund for Research under program 24. The Laboratoire du Rayonnement Electromagnetique is CNRS-CEA.
${ }^{1}$ S. J. Oh, J. W. Allen, and J. M. Lawrence, Phys. Rev. B 35, 2267 (1987).

${ }^{2}$ A. Kakizaki, H. Sugawara, I. Nagakura, Y. Ishikawa, Komatsubara, and T. Ishii, J. Phys. Soc. Jpn. 51, 2597 (1982).

${ }^{3}$ A. Rizzi, H. Moritz, and H. Luth, J. Vac. Sci. Technol. A 9, 912 (1991).

${ }^{4}$ M. C. Bost and J. E. Mahan, J. Appl. Phys. 58, 2696 (1985); 64, 2034 (1988).

5 J. Derrien, J. Chevrier, V. LeThanh, and J. E. Mahan, Appl. Surf. Sci. 56-58, 382 (1992).

${ }^{6}$ N. Onda, J. Henz, E. Muller, and H. von Kanel, Helv. Phys. Acta 64, 197 (1991); Appl. Surf. Sci. 56-58, 421 (1992).

${ }^{7}$ A. L. Vazquez de Parga, J. De la Figuera, C. Ocal, and R. Miranda, Europhys. Lett. 18, 585 (1992).

${ }^{8}$ N. E. Christensen, Phys. Rev. B 42, 7148 (1990).

${ }^{9}$ M. De Crescenzi, G. Gaggiotti, N. Motta, F. Patella, A. Balzarotti, and J. Derrien, Phys. Rev. B 42, 5871 (1990).

${ }^{10}$ Y. L. Gavriljuk, L. Y. Kachanova, and V. G. Lifshits, Surf. Sci. Lett. 256, L589 (1991); T. Urano and T. Kanaji; Appl. Surf. Sci. 33/34, 68 (1988).

${ }^{11}$ B. Egert and G. Panzner, Phys. Rev. B 29, 2091 (1984).

${ }^{12}$ J. Alvarez, J. Hinarejos, E. Michel, G. R. Castro, and R. Miranda, Phys. Rev. B 45, 14042 (1992).

${ }^{13}$ J. J. Jia, T. A. Callcott, W. L. O'Brien, Q. Y. Dong, D. R. Mueller, D. L. Ederer, Z. Tan, and J. I. Budnick, Phys. Rev. B 46, 9446 (1992); Ch. Aron, P. Auric, and C. Jeandey, Solid
State Commun. 79, 217 (1991).

${ }^{14}$ N. Jedrecy, Y. Zheng, A. Waldhauer, M. Sauvage-Simkin, and R. Pinchaux, Phys. Rev. B (to be published).

${ }^{15}$ W. Speier, E. v. Leuken, J. C. Fuggle, D. D. Sarma, L. Kumar, B. Dauth, and K. H. Bushow, Phys. Rev. B 39, 6008 (1989); G. Rossi, Surf. Sci. Rep. 7, 1 (1987).

${ }^{16}$ C. Calandra, O. Bisi, and G. Ottaviani, Surf. Sci. Rep. 4, 271 (1984).

${ }^{17}$ D. R. Hamann, Phys. Rev. Lett. 60, 313 (1988).

${ }^{18}$ M. Kohgi and Y. Ishikawa, Solid State Commun. 37, 833 (1981); O. Nakanishi, A. Yanase, and A. Hasegawa, J. Magn. Magn. Mater. 15-18, 879 (1980).

${ }^{19}$ J. F. van Acker, Z. M. Stadnik, J. C. Fuggle, H. Hoekstra, K. Bushow, and G. Stroink, Phys. Rev. B 37, 6827 (1988).

${ }^{20}$ C. Carbone, J. Kachel, R. Rochow, and W. Gudat, Z. Phys. B 79, 325 (1990); F. U. Hillebrecht, R. Jungblut, and E. Kisker, Phys. Rev. Lett. 65, 2450 (1990).

${ }^{21}$ C. Carbone and E. Kisker, Solid State Commun. 65, 1107 (1988).

${ }^{22}$ B. Sinkovic, P. D. Johnson, N. B. Brookes, A. Klarke, and N. V. Smith, Phys. Rev. Lett. 65, 1647 (1990).

${ }^{23}$ G. van der Laan, M. Surman, M. A. Hoyland, C. F. Flipse, B. T. Thole, H. Ogasawara, Y. Seiono, and A. Kotani, Phys. Rev. B 46, 9336 (1992).

${ }^{24}$ S. Doniach and M. Sunjic, J. Phys. C 3, 285 (1970).

${ }^{25}$ Y. Desausoy, J. Protas, R. Wandji, and B. Roques, Acta Crys- 
tallogr. Sec. B 27 (1971).

${ }^{26}$ T. Miller, T. C. Hsieh, and T. C. Chiang, Phys. Rev. B 33, 6983 (1986).

${ }^{27}$ G. Rossi, P. Roubin, D. Chandesris, and J. Lecante, Surf. Sci. 168, 787 (1986).
${ }^{28}$ G. Rossi, R. Jaeger, J. Stöhr, T. Kendelewicz, and I. Lindau, Phys. Rev. B 27, 5154 (1983).

${ }^{29}$ B. T. Thole and G. van der Laan, Phys. Rev. B 38, 3158 (1988). 